\title{
A 10 años del primer número de Mundo Nano. Un breve diagnóstico
}

\section{0 years after the first issue of Mundo Nano. A brief diagnosis}

\author{
Rogelio López Torres* \\ Editor asociado
}

El primer número de Mundo Nano. Revista Interdisciplinaria en Nanociencias y Nanotecnología se publicó en noviembre de 2008 y, con esta entrega, correspondiente al volumen 11 , número 21 , julio-diciembre de 2018, nuestra revista cumple 10 años. Mundo Nano es una publicación de la Universidad Nacional Autónoma de México (UNAM) que se va consolidando y constituye un referente importante en México. Surge por iniciativa de tres entidades académicas de la UNAM: el Centro de Investigaciones Interdisciplinarias en Ciencias y Humanidades, el Centro de Ciencias Aplicadas y Desarrollo Tecnológico hoy Instituto de Ciencias Aplicadas y Tecnología y el Centro de Nanociencias y Nanotecnología. Es un proyecto editorial que cuenta con el apoyo tanto de la Coordinación de la Investigación Científica como de la Coordinación de Humanidades de la UNAM, lo cual lo hace un ejercicio con clara vocación de vincular las ciencias y las humanidades, premisa fundamental en el abordaje interdisciplinario.

La importancia de esta enfoque epistémico y metodológico adquiere relevancia cuando surgen y se desarrollan tecnologías novedosas como la nanotecnología, que descubre, diseña, y produce nuevos materiales y procesos a escala nanométrica con características únicas. Estos materiales nanoestructurados o procesos nanohabilitados están revolucionando una gran cantidad de industrias en el mundo entero. Datos de Statnano precisan que, en 52 países operan 1,438 empresas con 7,286 productos nanohabilitados (product.statnano.com). Según Statnano, el grueso de productos NyN están en el mercado de EUA, seguido de lejos por el de China, Alemania, Suiza, Japón, Corea del Sur y Reino Unido. La mayoría de productos refiere a electrónicos, seguidos de aquellos para uso médico (sobre todo fármacos), cosméticos, materiales de construcción, aplicaciones medioambientales (sobre todo para el tratamiento y filtración de agua), de uso en la industria automotriz y en el avance de las energías renovables (product.statnano.com). En menor medida en alimentos, textiles, industria petrolera, electrodomésticos, agricultura, productos deportivos y de impresión.

* Universidad Nacional Autónoma de México, Centro de Investigaciones Interdisciplinrias en Ciencias y Humanidades, Ciudad de México, México. 
De cara a tal avance, se ha considerado por múltiples especialistas que México no puede rezagarse, de ahí que requiera impulsar el crecimiento y desarrollo de estas áreas del conocimiento. No obstante, a la par del estímulo, también es preciso conocer y evaluar el impacto y las implicaciones de esta tecnología en la sociedad; por ejemplo, en la economía, la salud y el medio ambiente, pero también en la propia construcción y avance del conocimiento y de la ética. Mundo Nano ha representado, en este sentido, un papel importante en la difusión del conocimiento y en la comunicación entre ciencia y sociedad.

Ya en el editorial del primer número se apuntaban los objetivos generales de la revista. Desde entonces, una de las primeras publicaciones en español que,

[...] surge de la inminente necesidad de impulsar un diálogo interdisciplinario de calidad sobre los avances reales, promesas e implicaciones de la nanotecnología con el objetivo de enriquecer la toma de decisiones nacionales referentes a la maximización y distribución de beneficios, la definición de responsabilidades y la minimización de costos innecesarios o indeseados. Mundo Nano busca colocarse en la vanguardia del debate científico-social al presentar avances y resultados de investigación de calidad de especialistas provenientes de las ciencias exactas, las ciencias sociales y las humanidades, del sector empresarial, de la esfera política [...]

Durante estos 10 años la revista se ha constituido en un valioso foro de análisis y discusión de las investigaciones en nanociencias y nanotecnología desarrolladas en las instituciones de educación superior y de investigación a lo largo y ancho del país, así como en otras latitudes. En ese sentido, es importante destacar también la colaboración de colegas e instituciones de otras regiones del mundo con las que hemos trabajado en torno a números especiales dedicados diversas temáticas. La revista publica artículos originales, dictaminados por pares académicos especialistas en la diversidad de aproximaciones y áreas de conocimiento que entrecruza "lo nano". Hemos publi-

FIGURA 1. Artículos y autores en diez años y 16 números de Mundo Nano.

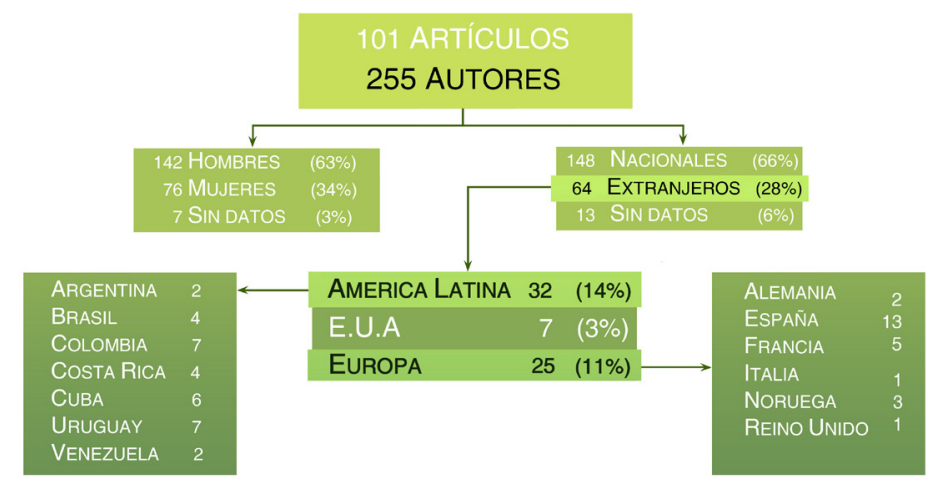

Fuente: Mundo $\mathrm{Nano}^{\circledR}$. 
FIGURA 2. Número de artículos y autores según el lugar de origen de su adscripción institucional.

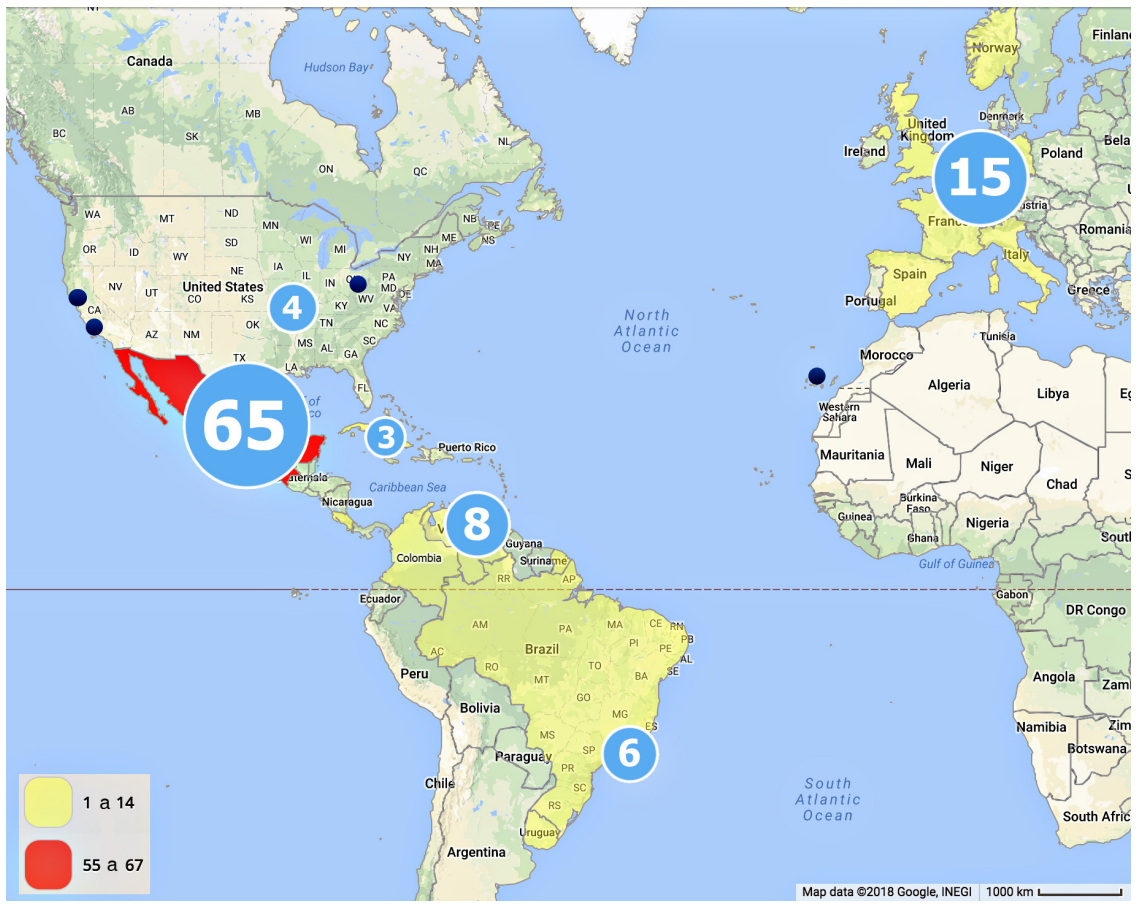

Fuente: Mundo Nano ${ }^{\circledR}$. Los colores muestran la intensidad de autores por país mientras que los círculos azules indican el número de artículos.

cado 101 artículos de 225 autores (en 16 números de la revista), tanto de instituciones mexicanas, como de América Latina, EUA y de países europeos (véanse las figuras 1 y 2).

Los problemas que han sido analizados en las páginas de la revista incluyen temas como el agua, energía, nanomedicina y nanobiología, nanoética, fármacos, nanotoxicidad, patentes, aspectos de la gobernanza y ética, divulgación de la nanociencia, de los lineamientos y la normatividad de la nanotecnología, el desarrollo de las nanociencias y nanotecnología en Argentina, Brasil, Cuba y España, entre otras temáticas (figura 3a y 3b).

También hemos publicado, en dos números, el "Catálogo nacional de instituciones de investigación con actividades en nanociencias y nanotecnología" (vol. 9, núm. 16, enero-junio y núm. 17, julio-diciembre 2016). Para la integración de este catálogo, atendieron la convocatoria de Mundo Nano 28 sedes de educación superior, del sistema de centros públicos de investigación del CONACyT y organismos descentralizados, distribuidos en 13 entidades federativas, en este catálogo colaboraron 72 personas, 24 mujeres y 48 hombres. La pertinencia de elaborar este mapa nacional, que da cuenta del estado del arte de la investigación y de las actividades docentes (en licenciatura 
Mundo Nano | www.mundonano.unam.mx

11(21), 7-11, julio-diciembre 2018 | http://dx.doi.org/10.22201/ceiich.24485691e.2018.21

Rogelio López Torres

FIGURA 3. Temáticas más abordadas en Mundo Nano.

$3 a$

\section{nanocompositos metálicos citotoxicidad}

compositos vidrio bioactivo protegiendo fármaco acarreadores

educación

nanobioética fibonacci

fotoimpresión

carbono multicapa

efecto teratogénico

carbón

arsénico

aplicaciones comercialización casimir

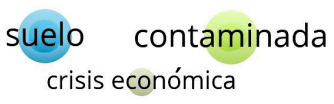

galio

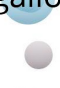

biomolecula

cápsula

nanoética

tecnología

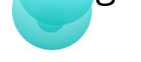

desarrollo

catálisis heterogeneizada

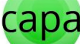

complejidad

divulgación

catodoluminiscencia

ubosoma

evaluación electroquímica

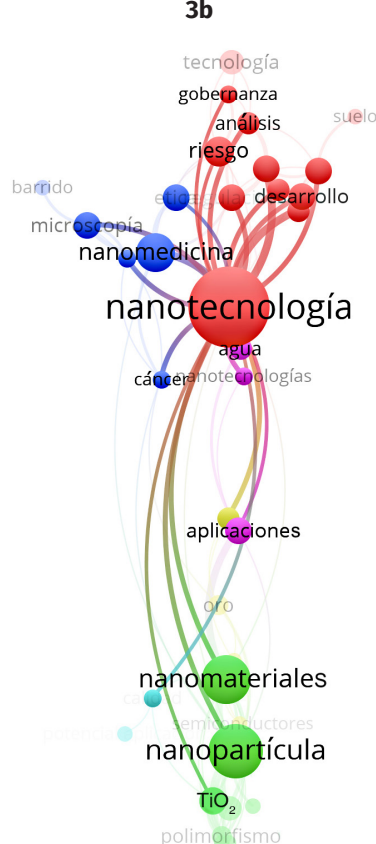

Fuente: Mundo $\mathrm{Nano}^{\circledR}$. La figura 3a muestra las temáticas más abordadas según las palabras clave de los artículos publicados en diez años. La figura 3b muestra la frecuencia y grados de asociación de tales palabras clave.

y posgrado) de las nanociencias y la nanotecnología, constituye un elemento fundamental de diagnóstico que permite orientar la generación de políticas públicas e institucionales para impulsar el desarrollo en estas áreas del conocimiento. Actualmente y derivado de tal esfuerzo se prepara un libro que conformará el tercer diagnóstico nacional sobre la NyN en México. ${ }^{1}$

Mundo Nano también ha sido foro para redes como la Red Internacional 'José Roberto Leite' de Divulgación y Formación en Nanotecnología-NANODYF (volumen 4, número 2, julio-diciembre de 2011), así como de sociedades científicas, la Academia Mexicana de Catálisis fue editora invitada del

${ }^{1}$ Previamente, otros esfuerzos se han realizado. En 2008, la Secretaría de Economía y el Centro de Investigaciones en Materiales Avanzados del conACyT publicaron el Diagnóstico y Prospectiva de la Nanotecnología en México. En 2014, la Red Temática de Nanociencias y Nanotecología (Red NyN) del CONACyT, en alianza con Axis elaboraron la Iniciativa para el desarrollo de la nanotecnología en México la cual se soportó en un análisis situacional de las nanociencias y la nanotecnología en México cuyo cierre de análisis fue noviembre de 2013. El consorcio institucional detrás de Mundo Nano, denominado nanounAM, se suma a estos esfuerzos con la publicación, en 2018, de Nanociencia y Nanotecnología en México: un balance de país. 
número 18 (enero-junio, 2017), dedicado justamente a analizar la investigación en catálisis en México y sus aplicaciones nano. En esa ocasión participaron 12 sedes académicas y de investigación y colaboraron 21 personas, 10 mujeres y 11 hombres de 5 entidades federativas. El Comité Editorial se ha fortalecido con la incorporación de nuevos colaboradores; también se ha integrado un Comité Científico que reúne entre ambos a 31 especialistas de diversas disciplinas, 6 mujeres y 25 hombres, con sede en 6 países.

En suma, podemos decir que, si bien son tres entidades universitarias las editoras de Mundo Nano, la creciente y valiosa participación de la comunidad científica nacional de las nanociencias y la nanotecnología ha sido quien en lo concreto ha enriquecido y fortalecido este proyecto editorial. A todos y todas, estamos agradecidos.

Aún queda mucho por hacer, especialmente de gestión editorial. En Mundo Nano estamos comprometidos en atender y cumplir nuevas disposiciones y requerimientos para mejorar día a día el posicionamiento y la proyección de la revista, aunque también es importante señalar que esta publicación ha construido un perfil propio, delineado por la producción científica: publicamos artículos originales de alta calidad académica sometidos a revisión por pares especializados, básicamente en el mundo de habla hispana. Se trata de una revista que ha tenido una importante función como training journal, pero también en la difusión del conocimiento interdisciplinar acerca del mundo nano.

Con este número se cierra su ciclo en versión impresa (aunque seguirá estando disponible bajo demanda) la cual tuvo un tiraje variante entre $500 \mathrm{y}$ 300 ejemplares, muchos de los cuales se han distribuido gratuitamente a bibliotecas de universidades públicas y privadas, y centros de investigación interesados. En su formato digital, el cual en su nuevo ciclo será ampliamente mejorado, no ha visto más que aumentar sus visitas. Tan sólo de 2011 hasta 2015, el sitio de la revista fue visto en más de 43 mil ocasiones (aproximadamente), lo que equivale a más de 700 visitas mensuales, provenientes de México y otras partes del mundo. Entre los artículos más descargados destaca "Metodologías para la síntesis de nanopartículas: controlando forma y tamaño" (volumen 5, 2012) con más de 12 mil descargas. Mejoraremos pues cuestiones de gestión interna, criterios de política editorial y de política para autores, revisores y lectores, manejo de metadatos y marcaje XML, indexaciones, código de ética y mecanismos de detección de plagio, entre otros aspectos.

Hemos cumplido 10 años y agradecemos a nuestros autores, revisores, y lectores, al Comité Editorial, editores científicos que han colaborado y colaboran hoy día con la revista, así como a las autoridades universitarias por su apoyo durante todo este tiempo, en especial a las coordinaciones de Humanidades y de la Investigación Científica, así como a la Subdirección de Revistas Académicas y Publicaciones Digitales, de la Dirección General de Publicaciones y Fomento Editorial de la UNAM. 